\title{
Vibrations of a columnar vortex in a trapped Bose-Einstein condensate
}

\author{
Lyndon Koens, ${ }^{1,2}$ Tapio P. Simula, ${ }^{3}$ and Andrew M. Martin ${ }^{1}$ \\ ${ }^{1}$ School of Physics, The University of Melbourne, Parkville, Victoria 3010, Australia \\ ${ }^{2}$ Department of Applied Mathematics and Theoretical Physics, University of Cambridge, Cambridge, CB3 OWA, United Kingdom \\ ${ }^{3}$ School of Physics, Monash University, Clayton, Victoria 3800, Australia
}

(Received 8 August 2012; published 14 June 2013)

\begin{abstract}
We derive a governing equation for a Kelvin wave supported on a vortex line in a Bose-Einstein condensate, in a rotating cylindrically symmetric parabolic trap, where it is assumed that the shape of the vortex line is dominated by the properties of the condensate at the center of the trap. From this solution the Kelvin wave dispersion relation is determined. In the limit of an oblate trap and in the absence of longitudinal trapping our results are consistent with previous work. We show that the derived Kelvin wave dispersion in the general case is in quantitative agreement with numerical calculations of the Bogoliubov spectrum.
\end{abstract}

DOI: 10.1103/PhysRevA.87.063614 PACS number(s): 67.85.De, 03.65.-w, 03.75.Lm, 05.30.Jp

\section{INTRODUCTION}

The behaviors of turbulent flows, tornadoes, mixing processes, synoptic-scale weather phenomena, and sunspots all critically depend on our understanding of vortex dynamics. Quantitative investigation of vortices started in the mid-1800s with the development of the Navier-Stokes equation, with the properties of a vortex being described by streamlines of vorticity. In 1880, Thomson (Lord Kelvin) determined the dispersion relation [1] for a specific excitation on a vortex line. This excitation takes the form of a chiral normal mode in which the perturbation propagates along a vortex line and rotates about its unperturbed position, distorting the vortex line into a helical shape.

In superfluids these vortices differ from their classical counterparts by having quantized circulation and a single line of vorticity (a vortex line) associated with them [2]. A quantized vortex line, in analogy with a classical vortex, supports Kelvin waves [2-11]. The first experimental investigations of Kelvin waves, in a superfluid, were carried out in cryogenically cooled helium [2-4,6]. More recently Bose-Einstein condensates (BECs) have provided a new platform to investigate the properties of quantized vortices [12-19]. The highly controllable nature of BEC systems has enabled the experimental investigation of Kelvin waves on a single vortex line [9]. The behavior of such waves has been investigated [20-22] and plays a crucial role in understanding the details of superfluid turbulence [23-25]. In trapped systems the Kelvin wave dispersion for a single vortex line has been obtained numerically, via solving the Bogoliubov spectrum for a single vortex line $[21,22,26]$. The Kelvin wave dispersion relation in the limit of long wavelengths and in the absence of trapping is [5]

$$
\omega=\frac{\hbar k^{2}}{2 M} \ln \left(\frac{1}{|k| r_{c}}\right),
$$

where $\omega$ is the excitation frequency of the mode, $k$ is the wave number, $M$ is the particle mass, and $r_{c}$, typically of the order of the healing length, is the vortex-core parameter.

A general formulation for a quantized vortex line in a trapped rotating BEC has proven difficult. Most methods have relied on matched asymptotic expansions [27-31]. Koens and Martin used such a procedure to obtain a set of equations that describe the behavior of a perturbed vortex line [29]. In this analysis the general equations [Eqs. (68) and (69) in Ref. [29]] contained an undetermined functional. Here we eliminate the functional to obtain a single equation that describes the radial position of a vortex line, supporting a Kelvin wave. We then determine the general solutions of this equation, enabling us to make favorable comparisons with previous results in limiting regimes [29,31-34]. From the general solution the Kelvin wave dispersion relation, for a single vortex line in a BEC in a cylindrically symmetric parabolic trap, is determined. This dispersion quantitatively agrees with numerically calculated Bogoliubov spectra [22], in contrast to previous analytic results [30].

\section{THEORY}

As shown in Ref. [29] the equations governing the positional dependence of the vortex line can be obtained. In cylindrical coordinates, defined by the radial $\hat{\rho}$, angular $\hat{\phi}$, and axial $\hat{z}$ unit vectors, these equations are

$$
\begin{aligned}
\frac{\partial \rho}{\partial t}= & -\frac{\hbar \rho\left[\ln \left(r_{c}\right)+1\right]}{2 M g\left|\Psi_{T F}\right|^{2}} \frac{\partial \phi}{\partial z} \frac{\partial V_{t r}}{\partial z}-\frac{\hbar\left[\ln \left(\frac{1}{r_{c}} \sqrt{\frac{\rho}{\rho\left(\partial_{z} \phi\right)^{2}-\partial_{z}^{2} \rho}}\right)+1\right]}{2 M} \\
& \times\left(\rho \frac{\partial^{2} \phi}{\partial z^{2}}+2 \frac{\partial \rho}{\partial z} \frac{\partial \phi}{\partial z}\right)-\frac{3 \hbar\left[\ln \left(\frac{R_{\perp}}{r_{c}}\right)+\frac{2}{3}\right]}{4 \rho M g\left|\Psi_{T F}\right|^{2}} \frac{\partial V_{t r}}{\partial \phi} \\
& +2 \frac{\Omega}{\rho \nabla_{\perp}^{2} V_{t r}} \frac{\partial V_{t r}}{\partial \phi}+\hat{\rho} \cdot \mathbf{E}\left(\frac{-\rho \frac{\partial \phi}{\partial z} \hat{\rho}+\frac{\partial \rho}{\partial z} \hat{\phi}}{g\left|\Psi_{T F}\right|^{2}} \frac{\partial V_{t r}}{\partial z}, \mathbf{r}\right),
\end{aligned}
$$

$$
\begin{aligned}
\rho \frac{\partial \phi}{\partial t}= & \frac{\hbar \ln \left(r_{c}\right)}{2 M g\left|\Psi_{T F}\right|^{2}} \frac{\partial \rho}{\partial z} \frac{\partial V_{t r}}{\partial z}+\frac{3 \hbar \ln \left(\frac{R_{\perp}}{r_{c}}\right)}{4 M g\left|\Psi_{T F}\right|^{2}} \frac{\partial V_{t r}}{\partial \rho} \\
& +\frac{\hbar \ln \left(\frac{1}{r_{c}} \sqrt{\frac{\rho}{\rho\left(\partial_{z} \phi\right)^{2}-\partial_{z}^{2} \rho}}\right)}{2 M}\left[\frac{\partial^{2} \rho}{\partial z^{2}}-\rho\left(\frac{\partial \phi}{\partial z}\right)^{2}\right] \\
& -2 \frac{\Omega}{\nabla_{\perp}^{2} V_{t r}} \frac{\partial V_{t r}}{\partial \rho}+\hat{\phi} \cdot \mathbf{E}\left(\frac{-\rho \frac{\partial \phi}{\partial z} \hat{\rho}+\frac{\partial \rho}{\partial z} \hat{\phi}}{g\left|\Psi_{T F}\right|^{2}} \frac{\partial V_{t r}}{\partial z}, \mathbf{r}\right),
\end{aligned}
$$


where $\rho$ and $\phi$ describe the radial and angular coordinates of the vortex line at a given $z$ (the dependence on $z$ is implicit), $V_{t r}$ is the trapping potential, $\left|\Psi_{T F}\right|^{2}$ is the Thomas-Fermi condensate density, $g$ is the interparticle interaction strength, $R_{\perp}$ is the radial Thomas-Fermi radius, $\Omega$ is the rotation frequency of the trap, and $\mathbf{E}$ is an unknown function that depends on the structure of the vortex line. Previous work has determined $\mathbf{E}$ by considering the case where the trapping and curving dependence in $\mathbf{E}$ can be treated separately; see Sec. VII of Ref. [29]. Specifically, two different scenarios were considered: one indicating a straight vortex's reaction to the trap confinement and one describing the behavior of wave perturbations on the line in the absence of confinement. Equations (2) and (3) were derived by noting that BECs with vortices within them have two natural length scales: the condensate length scale and the vortex-core radius. The full behavior of the system was then determined from the behavior of the condensate near to and far from the vortex core, via asymptotic expansion, separately, and ensuring that these two solutions matched in the overlapping region. In performing such an analysis to determine Eqs. (2) and (3) several approximations have been made. First, the wavelength of the excitation of the vortex line must be large, as it has to be a small perturbation from the central straight-line vortex. Second, for the poles of the condensate the derivation is invalidated as the radial size of the condensate becomes similar to the perturbation size (or the vortex-core size).

In general, Eqs. (2) and (3) relate the motion of the vortex line [left-hand sides (LHSs) of Eqs. (2) and (3)] to its distortion and coupling to the trapping potential [right-hand sides (RHSs) of Eqs. (2) and (3)]. E provides coupling between the motion of the vortex line and the trapping potential in the $z$ direction and therefore affect the first terms on the RHSs of Eqs. (2) and (3). Due to the unbalanced units of $\ln \left(r_{c}\right)$ of the first terms on the RHSs of Eqs. (2) and (3), E must take a form to balance this. Hence, these terms can be eliminated from each equation.

\section{RESULTS}

In this work, we are interested in the properties of helical waves in the presence of a harmonic trapping potential $V_{t r}\left(r_{\perp}=\rho, z\right)=M \omega_{\perp}^{2} \rho^{2} / 2+M \omega_{z}^{2} z^{2} / 2$, where $\omega_{\perp}$ and $\omega_{z}$ are the trapping frequencies in the $\rho$ and $z$ directions, respectively. Classically helical waves have $\phi$ structure of the form $\phi=k z-\omega t$. Assuming that $\phi$ has the same structure and that $\rho$ is time independent, the governing equation becomes

$$
\begin{aligned}
& {\left[\Omega-\omega-\frac{3 \hbar \omega_{\perp}^{2} \ln \left(\frac{R_{\perp}}{r_{c}}\right)}{4 g\left|\Psi_{T F}\right|^{2}}+\frac{\hbar k^{2} \Theta}{M}\right] \rho^{2}} \\
& =-\frac{\hbar \omega_{z}^{2} z \rho}{2 g\left|\Psi_{T F}\right|^{2}} \frac{\partial \rho}{\partial z}+\frac{\hbar \rho \Theta}{M} \frac{\partial^{2} \rho}{\partial z^{2}}-\frac{\hbar[2 \Theta+1]}{M}\left(\frac{\partial \rho}{\partial z}\right)^{2},
\end{aligned}
$$

where $\Theta=-\ln \left(r_{c} \sqrt{k^{2}-\partial_{z}^{2} \rho / \rho}\right) / 2$. The LHS of Eq. (4) defines the properties of a vortex line when $\rho$ is constant. The first of the terms on the RHS contains the influence from $z$ confinement and the other two represent the influence the curving of the vortex line in the radial direction has on its motion in $\phi$ and $\rho$, respectively.
Equation (4) does not admit an analytic solution in a closed form. However, by assuming that (i) $\partial_{z}^{2} \rho / \rho$ is small compared to $k^{2}$ and may be approximated by $\beta$ and (ii) taking the effect of $g\left|\Psi_{T F}\right|^{2}$ to be a constant, to leading order, defined by the condensate chemical potential $\mu=g\left|\Psi_{T F}(0,0)\right|^{2}$, Eq. (4) reduces to

$$
\gamma \rho^{\prime 2}=-z^{\prime} \rho^{\prime} \frac{\partial \rho^{\prime}}{\partial z^{\prime}}+\zeta \rho^{\prime} \frac{\partial^{2} \rho^{\prime}}{\partial z^{\prime 2}}-\varepsilon\left(\frac{\partial \rho^{\prime}}{\partial z^{\prime}}\right)^{2}
$$

where $\zeta=-\ln \left(\tilde{k}^{2}-\tilde{\beta}\right) / 4$, with $\tilde{k}=r_{c} k, \tilde{\beta}=r_{c}^{2} \beta$, and $\varepsilon=$ $1+2 \zeta$. In Eq. (5) $z^{\prime}=z / R_{z}$, with $R_{z}$ being the axial ThomasFermi radius, $\rho^{\prime}=\rho / r_{c}$,

$$
\gamma=\Lambda^{2 / 5}[\tilde{\Omega}-\tilde{\omega}]+\tilde{k}^{2} \zeta \Lambda^{2 / 5}-\frac{3}{10 \lambda^{2}} \ln \Lambda,
$$

where $\Lambda=\frac{15 a N \hbar^{2}}{M^{2} \lambda^{4} \omega_{\perp}^{2} r_{c}^{5}}$ parametrizes the interactions, with $a$ being the $s$-wave scattering length and $N$ the number of particles in the condensate, and $\lambda=\omega_{z} / \omega_{\perp} . \tilde{\Omega}=M r_{c}^{2} \Omega / \hbar$ and $\tilde{\omega}=M r_{c}^{2} \omega / \hbar$ are the dimensionless trap rotation and Kelvin wave frequency, respectively. The two assumptions made imply that (i) the curvature in the $z$ direction of the radial displacement of the vortex line is much smaller than the wave number and (ii) the condensate density is considered to be locally homogeneous. For the second assumption, although the condensate density is taken to be a constant where it was explicitly written in Eq. (4), this does not discard its influence. In the derivation of Eqs. (2) and (3) the influence of the changing condensate density is included. Specifically, it was assumed to have a Thomas-Fermi profile which can be written as the central chemical potential minus the trapping potential. Similarly, in Eq. (4) it is possible to express $\left|\Psi_{T F}\right|^{2}$ in terms of the chemical and trapping potentials. Hence where gradients of the profile are involved $\left|\Psi_{T F}\right|^{2}$ is replaced with gradients of the trapping potential. Since Eqs. (2) and (3) are most accurate away from the edges of the condensate it is thereby reasonable to assume that the reciprocal of $\left|\Psi_{T F}\right|^{2}$ is close to constant.

Making a change of variables such that $\rho^{\prime}\left(z^{\prime}\right)$ has the form $f\left(z^{\prime}\right)^{\zeta /(\varepsilon-\zeta)}$ Eq. (5) reduces to

$$
\gamma(\zeta-\varepsilon) f\left(z^{\prime}\right)+\zeta\left[z^{\prime} \frac{d f\left(z^{\prime}\right)}{d z^{\prime}}-\zeta \frac{d^{2} f\left(z^{\prime}\right)}{d z^{\prime 2}}\right]=0,
$$

with the continuum-limit solution

$\rho^{\prime}\left(z^{\prime}\right)=\left[C_{1} H_{A}\left(\frac{z^{\prime}}{\sqrt{2 \zeta}}\right)+C_{21} F_{1}\left(-\frac{A}{2} ; \frac{1}{2} ; \frac{z^{\prime 2}}{2 \zeta}\right)\right]^{-\zeta /(1+\zeta)}$,

where $A=\gamma(\varepsilon-\zeta) / \zeta, H_{p}(z)$ is the Hermite polynomial of order $p,{ }_{1} F_{1}(l ; m ; x)$ is the Kummer confluent hypergeometric function, and $C_{1}$ and $C_{2}$ are integration constants.

Equation (8) is the central result of this paper. As such it is prudent to reconsider its regimes of validity. Specifically, this solution is for the radial position of the vortex as a function of $z^{\prime}$, in a cylindrically symmetric parabolic trap. In obtaining this solution several approximations have been made, specifically as follows:

(a) The original governing equations (2) and (3) are derived from the behavior of the condensate near to and far from the vortex core, via asymptotic expansion, separately, and ensuring that these two solutions matched in the overlapping 
region. This imposes the conditions that the wavelength of the excitation of the vortex line must be large, as it has to be a small perturbation from the central straight-line vortex. Additionally, for the "top" and "bottom" of the condensate the governing equations are poor, as the radial size of the condensate becomes similar to the perturbation size.

(b) In determining Eq. (4) a specific form for the trapping potential was assumed: a cylindrically symmetric parabolic potential. Additionally, a specific structure for the vortex-line excitation was assumed: $\phi=k z-\omega t$. This imposes the constraint on the solution that $k$ is uniform throughout the condensate. Numerical simulations show this not to be true [22]; however, this assumption implies that the properties of the vortex line are primarily governed by the properties of the center of the condensate, where the density profile is approximately constant.

(c) To obtain Eq. (5) it was assumed that the curvature in the $z$ direction of the radial displacement of the vortex line is much smaller than the wave number and the condensate density is considered to be locally, with respect to the vortex line, homogeneous.

In summary these assumptions imply that Eq. (8) provides a description for the vortex-line shape where it is assumed that the shape is dominated by the properties of the condensate at the center of the trap.

Because of the axisymmetry of the trapping potential, the vortex-line structure described is physical when $\rho^{\prime}$ is not multivalued within the condensate radius $\left(z^{\prime}= \pm 1\right)$, and is symmetric or antisymmetric across $z^{\prime}=0$. Due to the use of cylindrical coordinates and the $\phi=k z-w t$ ansatz Eq. (8) cannot produce antisymmetric solutions. Equation (8) is single valued and symmetric if $C_{1}=0$, reducing the solution to the symmetric hyper-geometric function, or if $A$ is a positive even integer, making $H_{A}(x)$ symmetric. In general there is no physical reason to restrict $A$ to be an integer; hence we consider only the case where $C_{1}=0$. This solution gives $\rho^{\prime}$ a $U$ shape, centered around $z^{\prime}=0$; for example see Fig. 1(a).

From Eq. (8) it is possible to check the assumption $|\tilde{\beta}| / \tilde{k}^{2} \ll 1$. At $z^{\prime}=0$ this ratio is typically of order $10^{-7}$. Nevertheless $\tilde{\beta}$ does depend on the ratio of the trapping frequencies, $\lambda$, with $\tilde{\beta} \rightarrow 0$ as $\lambda \rightarrow \infty$ and $\tilde{\beta} \rightarrow-\infty$ as $\lambda \rightarrow 0$. The divergence in $\tilde{\beta}$ as $\lambda \rightarrow 0$ is slow, indicating that $\tilde{\beta}$ plays a relatively insignificant role in Eq. (4) unless the trap is extremely prolate. In general, we calculate $\tilde{\beta}$ self-consistently, through the definition $\tilde{\beta}=r_{c}^{2} \partial_{z}^{2} \rho / \rho$.

\section{A. Oblate limit}

Equation (8) simplifies in the limits of extremely oblate $(\lambda \rightarrow \infty)$ and prolate $(\lambda \rightarrow 0)$ trapping potentials. In the oblate limit, $\gamma \rightarrow 0, \zeta \rightarrow-\ln (|\tilde{k}|) / 2$, and $\varepsilon \rightarrow 1-\ln (|\tilde{k}|)$, Eq. (5) has the solution

$$
\rho^{\prime}\left(z^{\prime}\right)=\left[D_{1}(1+\zeta) \sqrt{2 \pi} \frac{\operatorname{erf}\left(\frac{i z^{\prime}}{\sqrt{2 \zeta}}\right)}{i}+2 \sqrt{\zeta} D_{2}\right]^{-\zeta /(1+\zeta)}
$$

where $D_{1}$ and $D_{2}$ are again integration constants and $\operatorname{erf}(x)$ is the error function. Applying the symmetry and divergence conditions $\rho^{\prime}=\left(2 \sqrt{\zeta} D_{2}\right)^{-\zeta /(1+\zeta)}$, if $\rho^{\prime}$ is finite, from Eq. (5)

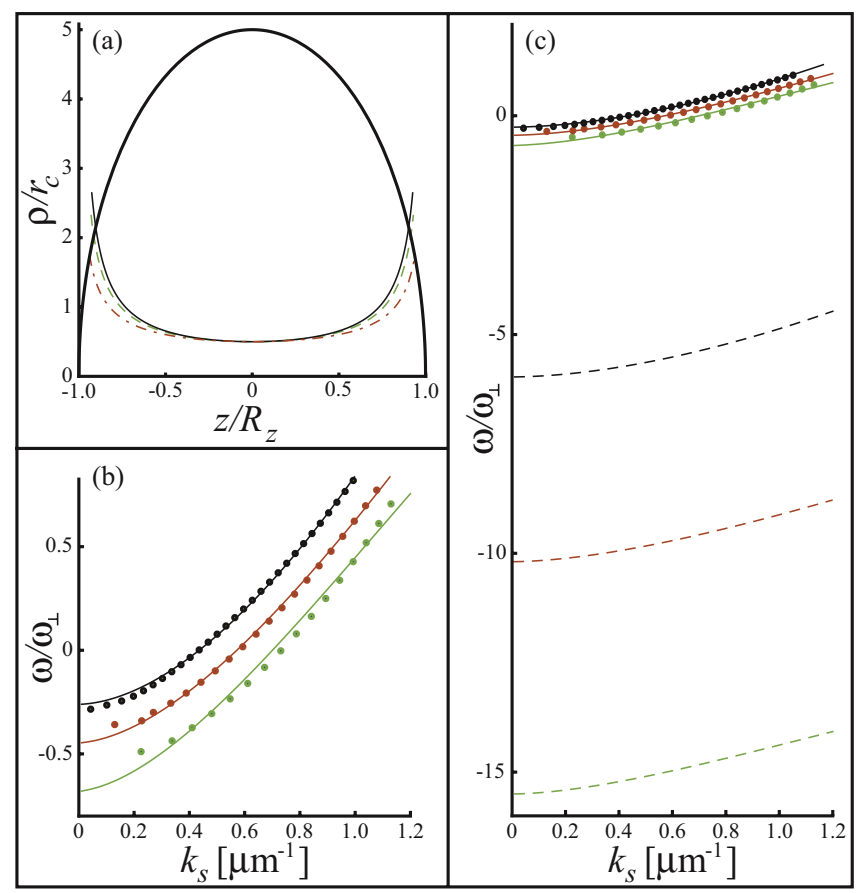

FIG. 1. (Color online) (a) The vortex-line shape in a prolate BEC, as given by Eq. (12), for $\tilde{k}=10^{-3}$ (black solid curve), $\tilde{k}=10^{-2}$ (green dashed curve), and $\tilde{k}=0.1$ (red dash-dotted curve). We have assumed $R_{\perp} / r_{c}=5, \sqrt{A / \zeta}=\pi / 2, F_{1}=0.5$, and $F_{2}=0$. The thick black solid line defines the Thomas-Fermi surface of the BEC. (b),(c) The dispersion relations for Kelvin waves, for $N=10^{4}$ (lower green realizations), $N=5 \times 10^{4}$ (middle red realizations), and $N=5 \times 10^{5}$ (upper black realizations) rubidium atoms in a harmonic trap: $\omega_{\perp}=2 \pi \times 98.5 \mathrm{rad} / \mathrm{s}$ and $\omega_{z}=2 \pi \times 11.8 \mathrm{rad} / \mathrm{s}$. In (b) and (c) the numerical calculations (dots) [22] and analytic results from Eq. (15) (solid curves) are plotted. In (c) the dashed curves show previous analytic results [30]. For the solid curves and dashed curves in (b) and (c) the following scaling parameters have been used for $r_{c}$ and $k: \alpha=0.134,0.257$, and 0.264 for $N=5 \times 10^{5}, 5 \times 10^{4}$, and $10^{4}$, respectively, and $s=\left(\lambda R_{z} / l_{\omega_{z}}\right)^{1 / 2}$ for all $N$.

the Kelvin wave has the frequency

$\omega_{2 D}=-\frac{3 \hbar \omega_{\perp}^{2} \ln \left(R_{\perp} / r_{c}\right)}{4 \mu}-\frac{\hbar k^{2} \ln \left(r_{c} \sqrt{k^{2}-\beta}\right)}{2 M}+\Omega$.

This is consistent with previous work $[31,32]$ which shows that an off-centered vortex in an oblate condensate is straight and has a precession frequency $\Omega-3 \hbar \omega_{\perp}^{2} \ln \left(R_{\perp} / r_{c}\right) / 4 \mu$, in the limit $k \rightarrow 0$.

\section{B. Prolate limit}

In the prolate limit $(\lambda \rightarrow 0), \gamma, \zeta$, and $\varepsilon$ all tend to $\infty$. Hence Eq. (5) becomes

$$
\rho^{\prime 2}=\frac{\zeta}{\gamma} \rho^{\prime} \frac{\partial^{2} \rho^{\prime}}{\partial z^{\prime 2}}-\frac{\varepsilon}{\gamma}\left(\frac{\partial \rho^{\prime}}{\partial z^{\prime}}\right)^{2}
$$

with the solution

$$
\rho^{\prime}\left(z^{\prime}\right)=F_{1}\left\{\cos \left[\left(z^{\prime}-\zeta F_{2}\right) \sqrt{\frac{A}{\zeta}}\right]\right\}^{-\zeta /(1+\zeta)} .
$$


Again $\rho^{\prime}$ has only a symmetric solution $\left(F_{2}=0\right)$. This causes $\rho^{\prime}$ to have a $1 / \cos (z)$-like structure similar to that predicted in simulations [33,34]. Typical solutions of Eq. (12) are plotted in Fig. 1(a), demonstrating the $U$ shape of the radial coordinate. Furthermore, as $-\frac{\zeta}{1+\zeta}$ depends on $k$, this $U$ shape will widen as the momentum of the wave increases, as seen in Fig. 1(a). At low $\tilde{k}$, the line takes an almost inverted cosine shape and becomes straight as $\tilde{k} \rightarrow 1$.

To obtain the dispersion relation for the prolate case Eq. (12) needs to be single valued within the condensate. This gives the condition $\sqrt{A / \zeta}=\pi / 2$ or

$$
\omega=\omega_{2 D}-\frac{\hbar \omega_{z}^{2} \pi^{2} \zeta^{2}}{8 \mu(1+\zeta)},
$$

which is an approximation to the dispersion relation of helical waves in a prolate trap, and is distinct from the solution for $\omega_{z} \equiv 0$. This occurs because any trapping in $z$ provides a scale over which the vortex line can curve. Hence, when there is no trapping in $z, \varepsilon / \gamma=\zeta / \gamma=0$, turning Eq. (5) into $\gamma \rho^{\prime 2}=0$. Therefore, for the case of $\omega_{z}=0$ the dispersion relation is the same as in the oblate limit, given by Eq. (10), i.e., the vortex line is straight [29,31,32].

\section{General solution}

To determine the general dispersion relation from Eq. (8) a similar treatment to that for the extremely prolate trap is employed. As the $\rho^{\prime}$ solution is a hypergeometric function to a negative power, the zeros of the hypergeometric function must lie outside the condensate. The zeros of the confluent hypergeometric function ${ }_{1} F_{1}(l ; m ; x)$ can be approximated by [35]

$$
X_{0} \approx \frac{\pi^{2}\left(r+\frac{m}{2}-\frac{3}{4}\right)^{2}}{2 m-4 l},
$$

where $X_{0}$ is the approximate $x$ value of the $r$ th zero. Combining Eq. (14) with the form of the hypergeometric function in Eq. (8) and stipulating that the first zero is at $z^{\prime}=1$ the dispersion relation becomes

$$
\omega=\omega_{2 D}+\frac{\hbar \omega_{z}^{2} \zeta\left(2-\pi^{2} \zeta\right)}{8 \mu(1+\zeta)},
$$

or equivalently, for $\beta / k^{2} \rightarrow 0$,

$$
\omega=\omega_{0}+\omega_{1}+\frac{\hbar k^{2} \ln \left(\frac{1}{r_{c}|k|}\right)}{2 M}+\Omega,
$$

where

$$
\begin{aligned}
& \omega_{0}=-\frac{3 \hbar \omega_{\perp}^{2} \ln \left(\frac{R_{\perp}}{r_{c}}\right)}{4 \mu} \\
& \omega_{1}=-\frac{\hbar \omega_{z}^{2} \ln \left(r_{c}|k|\right)\left[4+\pi^{2} \ln \left(r_{c}|k|\right)\right]}{16 \mu\left[2-\ln \left(r_{c}|k|\right)\right]} .
\end{aligned}
$$

Equations (15) and (16) indicate that helical waves, in a parabolic trap, obey the usual dispersion relation [Eq. (1)], with two constants $\omega_{0}$, from confinement in $\rho$, and $\omega_{1}$, from confinement in $z$. The form of $\omega_{0}$ matches that for extremely oblate traps [Eq. (10)], while $\omega_{1}$ contains new behavior. Essentially, $\omega_{1}$ is constant, with weak logarithmic dependence on $k$, and becomes larger as $\omega_{z}$ increases. As $\lambda \rightarrow \infty$ Eq. (15) does not replicate the dispersion in the oblate trapping limit. This is because in the very oblate limit Eq. (14) fails to predict the location of the zeros accurately.

\section{COMPARISON WITH BOGOLIUBOV SPECTRA}

To test the validity of the above analysis we now compare the solutions of Eq. (15) with numerical calculations [22]. The dispersion relations of Kelvin waves in a prolate condensate for different particle numbers corresponding to numerical calculations [22] are shown in Figs. 1(b) and 1(c) (dots). Previous analytic predictions in this limit [see Eq. (70) in Ref. [30]] poorly replicated these results [dashed curves in Fig. 1(c)].

To compare these numerical results with Eq. (15), $r_{c}$ needs to first be considered. The core parameter $r_{c}$, implicit in Eq. (15), characterizes the vortex-core size. In a trapped BEC the healing length $\xi$ is of the order of the vortex-core radius, which is a function of position. As such we define the core parameter $r_{c}=\alpha \bar{\xi}$ to be some fraction $\alpha$ of the healing length averaged over the Thomas-Fermi volume: $\bar{\xi}=\left(R_{\perp}^{2} R_{z} / 6 a N\right)^{1 / 2}$. To compare with numerical results we allow $\alpha$ to be a free parameter, of order 1 .

In Figs. 1(b) and 1(c) (solid curves) we plot the Kelvin wave dispersion, Eq. (15), where we have rescaled $k \rightarrow s k=k_{s}$. Due to the inhomogeneity of the condensate we expect $k$ to vary spatially. As such this rescaling is motivated by the observation [22] that in numerical calculations $k$ varies along the vortex line. Interestingly, we find that the matching between analytical results and numerical calculations is optimized for $s=\left(\lambda R_{z} / l_{\omega_{z}}\right)^{1 / 2}$, where $l_{\omega_{z}}=\sqrt{\hbar /\left(m \omega_{z}\right)}$ is the harmonic oscillator length in $z$.

Figure. 1(c) shows that Eq. (15) (solid curves) is much closer to the numerical results than the previous analytic predictions (dashed curves) [30]. Previous calculations assumed that the confinement in $z$ strongly affected the precession frequency while our analysis creates only a small correction. This relates to the fact that Ref. [30] assumed that the influence on the vortex precession of the trap was the same perpendicular and parallel to the line's direction. Our work shows that it is still possible to solve the the problem without making this simplification. As a result we find that the influence on the vortex precession from the trap is not the same perpendicular and parallel to the line's direction. Additionally, Fig. 1(b) shows excellent quantitative agreement between the numerical and analytical results, with the agreement improving as the number of particles increases and the BEC becomes more Thomas-Fermi-like. The general features of the dispersion relation are dominated by (i) a frequency shift and (ii) a functional form proportional to $-k^{2} \ln r_{c} k$. The shift is dominated by the confinement in $\rho$, given by $\omega_{0}$, with a small contribution from the confinement in $z$, given by the second term in Eq. (15). The functional dependence of $\omega$ is essentially dominated by the solution for a vortex line in an untrapped condensate, Eq. (1), also with a small correction arising from the confinement in $z$, given by the second term in Eq. (15). We note that, in the context of Fig. 1, the self-consistent determination of $\beta$ influences the dispersion only for $k<10^{-4}$ $\mu \mathrm{m}^{-1}$ and hence, in Figs. 1(b) and 1(c), plays no role over the wave vectors considered. 


\section{CONCLUSIONS}

In summary, we have analytically determined the Kelvin wave dispersion relation for a vortex line in a BEC trapped in a cylindrically symmetric parabolic trap, Eq. (15), where it is assumed that the shape of the vortex line is dominated by the properties of the condensate at the center of the trap. This result quantitatively agrees with the numerical calculations, in contrast to previous descriptions. We also find that the dispersion relation in the oblate trapping limit, Eq. (10), coincides with previous analytical work. In general these results are derived from the governing equation of motion for
Kelvin waves on a quantized vortex line, Eq. (5). Equation (5) successfully predicts the behavior in very oblate and prolate condensates, and mathematically shows the link between the vortex-line shape with no trapping in $z$ and strong trapping in $z$. Within the context of experimental activity in the study of excitations of quantized vortex lines in trapped BECs $[9,15,36,37]$, this work provides a simple analytic tool for the analysis of Kelvin waves. Additionally, given the close agreement between numerical calculations and Eq. (15), we expect future experimental measurements of the Kelvin wave spectra to be in close agreement with our analytical description.
[1] W. L. K. Thomson, Philos. Mag. 10, 155 (1880).

[2] R. Donnelly, Quantized Vortices in Helium II (Cambridge University Press, Cambridge, 1991).

[3] H. Hall, Proc. R. Soc. London, Ser. A. 245, 546 (1958).

[4] E. Andronikashvili and D. Tsakadze, Sov. Phys. JETP 10, 227 (1960).

[5] L. P. Pitaevskii, Sov. Phys. JETP 13, 451 (1961).

[6] R. A. Ashton and W. I. Glaberson, Phys. Rev. Lett. 42, 1062 (1979).

[7] E. Sonin, Rev. Mod. Phys. 59, 87 (1987).

[8] R. Epstein and G. Baym, Astrophys. J. 387, 276 (1992).

[9] V. Bretin, P. Rosenbusch, F. Chevy, G. V. Shlyapnikov, and J. Dalibard, Phys. Rev. Lett. 90, 100403 (2003).

[10] A. L. Fetter, Phys. Rev. A 69, 043617 (2004).

[11] A. L. Fetter, Rev. Mod. Phys. 81, 647 (2009).

[12] M. R. Matthews, B. P. Anderson, P. C. Haljan, D. S. Hall, C. E. Wieman, and E. A. Cornell, Phys. Rev. Lett. 83, 2498 (1999).

[13] B. P. Anderson, P. C. Haljan, C. E. Wieman, and E. A. Cornell, Phys. Rev. Lett. 85, 2857 (2000).

[14] K. W. Madison, F. Chevy, W. Wohlleben, and J. Dalibard, Phys. Rev. Lett. 84, 806 (2000).

[15] F. Chevy, K. W. Madison, and J. Dalibard, Phys. Rev. Lett. 85, 2223 (2000).

[16] J. V. J. R. Abo-Shaeer, C. Raman, and W. Ketterle, Science 292, 476 (2001).

[17] E. Hodby, G. Hechenblaikner, S. A. Hopkins, O. M. Maragó, and C. J. Foot, Phys. Rev. Lett. 88, 010405 (2001).

[18] B. Anderson, J. Low. Temp. Phys. 161, 574 (2010).

[19] N. G. Parker, R. M. W. van Bijnen, and A. M. Martin, Phys. Rev. A 73, 061603(R) (2006).
[20] T. Mizushima, M. Ichioka, and K. Machida, Phys. Rev. Lett. 90, 180401 (2003).

[21] T. P. Simula, T. Mizushima, and K. Machida, Phys. Rev. Lett. 101, 020402 (2008).

[22] T. P. Simula, T. Mizushima, and K. Machida, Phys. Rev. A 78, 053604 (2008).

[23] E. Kozik and B. Svistunov, Phys. Rev. Lett. 92, 035301 (2004).

[24] W. Vinen, J. Low Temp. Phys. 145, 7 (2006).

[25] W. Halperin and M. Tsubota, Progress in Low Temperature Physics: Quantum Turbulence (Elsevier Science, Amsterdam, 2008).

[26] T. Isoshima and K. Machida, J. Phys. Soc. Jpn. 66, 3502 (1997).

[27] L. M. Pismen and J. Rubinstein, Physica D 47, 353 (1991).

[28] B. Y. Rubinstein and L. M. Pismen, Physica D 78, 1 (1994).

[29] L. Koens and A. M. Martin, Phys. Rev. A 86, 013605 (2012).

[30] A. A. Svidzinsky and A. L. Fetter, Phys. Rev. A 62, 063617 (2000).

[31] A. A. Svidzinsky and A. L. Fetter, Phys. Rev. Lett. 84, 5919 (2000).

[32] S. A. McGee and M. J. Holland, Phys. Rev. A 63, 043608 (2001).

[33] A. Aftalion and T. Riviere, Phys. Rev. A 64, 043611 (2001).

[34] J. J. García-Ripoll and V. M. Pérez-García, Phys. Rev. A 64, 053611 (2001).

[35] NIST Handbook of Mathematical Functions, edited by F. W. J. Olver, D. W. Lozier, R. F. Boisvert, and C. W. Clark (Cambridge University Press/NIST, Cambridge/New York, 2010).

[36] P. C. Haljan, B. P. Anderson, I. Coddington, and E. A. Cornell, Phys. Rev. Lett. 86, 2922 (2001).

[37] P. Rosenbusch, V. Bretin, and J. Dalibard, Phys. Rev. Lett. 89, 200403 (2002). 